\title{
Current-voltage characteristics of asymmetric double-barrier Josephson junctions
}

\author{
E.V. Bezuglyi ${ }^{\text {a }}$, E.N. Bratus' ${ }^{\text {a }}$, V.S. Shumeiko ${ }^{\text {b,* }}$ \\ a B.Verkin Institute for Low Temperature Physics and Engineering, National Academy of Sciences, 61103 Kharkov, Ukraine \\ ${ }^{\mathrm{b}}$ Department of Microtechnology and Nanoscience, Chalmers University of Technology, S-41296 Göteborg, Sweden
}

\section{A R T I C L E I N F O}

\section{Article history:}

Received 17 January 2014

Accepted 2 February 2014

Available online 10 February 2014

\section{Keywords:}

Josephson junction

Proximity minigap

Subharmonic gap structure

\begin{abstract}
A B S T R A C T
We develop a theory for the current-voltage characteristics of diffusive superconductor-normal metal-superconductor Josephson junctions with resistive interfaces and the distance between the electrodes smaller than the superconducting coherence length. The theory allows for a quantitative analytical and numerical analysis in the whole range of the interface transparencies and asymmetry. We focus on the regime of large interface resistance compared to the resistance of the normal region, when the electron-hole dephasing in the normal region is significant and the finite length of the junction plays a role. In the limit of strong asymmetry we find pronounced current structures at the combination subharmonics of $\Delta+\Delta_{g}$, where $\Delta_{g}$ is the proximity minigap in the normal region, in addition to the subharmonics of the energy gap $2 \Delta$ in the electrodes. In the limit of rather transparent interfaces, our theory recovers a known formula for the current in a short mesoscopic connector - a convolution of the current through a single-channel point contact with the transparency distribution for an asymmetric double-barrier potential.
\end{abstract}

(c) 2014 Elsevier B.V. All rights reserved.

\section{Introduction}

During last few years a large number of experimental researches has been done on the proximity effect in semiconductor nanowires connected to superconducting electrodes [1-7,9,8,10,11]. Hybrid devices of the nanowires have demonstrated Andreev subgap conductance, Josephson field effect, and Cooper-pair beam splitting. More recently, the nanowire hybrid devices attracted new attention following theoretical predictions of Majorana bound states in nanowire proximity structures.

From a theory viewpoint, the majority of investigated devices fall into the category of mesoscopic diffusive superconductornormal metal-superconductor (SNS) junctions with the length smaller or comparable to the superconducting coherence length. These devices typically have about 100 conducting channels, impurity mean free path $\sim 30-50 \mathrm{~nm}$, and the length varying from tens to hundreds nanometers, i.e. the Thouless energy $E_{\mathrm{Th}}$ in the range of $10-0.1 \mathrm{meV}$. This is larger or comparable to the energy gap $\Delta$ in superconducting $\mathrm{Al}$, which is used as the electrode material. The most interesting regime of a strong proximity effect, manifested by considerable Josephson current, is achieved in junctions

\footnotetext{
* Corresponding author. Tel.: +46 317723144.

E-mail address: vitaly.shumeiko@chalmers.se (V.S. Shumeiko).
}

with rather transparent nanowire-superconductor interfaces, whose transparencies typically exceed 0.1 .

The physics of the equilibrium proximity effect in such junctions is qualitatively well understood, and a quantitative theory for the dc Josephson transport has been developed by many authors on the basis of the quasiclassical Greens function technique, see, e.g. [12] and references therein.

Quantitative description of the ac Josephson effect is more challenging. The difficulty here arises from the presence of the time dependence of the dynamics in the normal region, in addition to the spatial inhomogeneity and nonlinearity. The problem was found solvable in diffusive point contacts [13,14], where an approximation of the zero contact length is appropriate. In that case, the problem can be reduced to the single channel coherent multiple Andreev reflection (MAR) problem [15-17]. Such an approximation is not suitable for the interpretation of the nanowire experiments, which show pronounced length dependence of the transport characteristics.

A step towards solving this problem was made in [18], where the coherent MAR problem has been analytically solved for a finite-length SNS junction with highly resistive interfaces (I), SINIS. In this model, the length of the junction is assumed to be small, but it cannot be put equal to zero because of significant dwell time of quasiparticles in the normal region confined by the strong interface barriers. As it was shown, the parameter that defines the short 
junction limit in this case is $\gamma=\left(R / R_{N}\right)\left(\Delta / E_{\mathrm{Th}}\right) \ll 1$, rather than $\Delta / E_{\mathrm{Th}} \ll 1$, where $R$ is the interface resistance and $R_{N}$ is the resistance of the normal region. Therefore even if the latter condition is fulfilled, the parameter $\gamma$ can be large, $\gamma \gg 1$. This is the most interesting regime, the physics of which is characterized, qualitatively similar to the long junction case, by large electron-hole dephasing in the normal region, leading to significant length dependence of the transport.

Solution of this problem is also important for understanding properties of the coherent current transport in planar $\mathrm{Nb} / \mathrm{Al}_{2} \mathrm{O}_{3} /$ $\mathrm{Al} / \mathrm{Al}_{2} \mathrm{O}_{3} / \mathrm{Nb}$ tunnel junctions which can be used as basic elements of practical superconducting electronics: rapid single flux quantum devices [19], voltage standards [20], high-frequency mixers [21], SQUIDs [22] (see also a review in [23]). As a rule, in such devices, the thickness of the Al layer is about $10 \mathrm{~nm}$, and the parameter $\gamma$ may achieve the values of the order of $10^{2}-10^{4}$.

In this paper we discuss the extension of theory developed in [18] to a practically important case of asymmetric junctions, namely junctions with different interface resistances. As we show, the asymmetry leads to a qualitative change of the IVC characteristics. In these junctions a novel set of current features appears at subharmonics of $\Delta+\Delta_{g}$, where $\Delta_{g}$ is the proximity induced minigap in the normal region.

The structure of the paper is as follows. A formal solution for the Keldysh-Green's function equation is presented in Section 2. Section 3 is devoted to demonstration of computation of equilibrium Josephson current using the found solution. In Section 4, a general non-equilibrium case is considered, and the dc currentvoltage characteristics are computed in Section 5; there we present the numerical results and analytical expressions for the partial MAR currents and the excess current.

\section{Construction of approximate solution}

We describe our junction with the diffusive equation [24] for the Keldysh-Green's function $\breve{G}\left(x, t_{1}, t_{2}\right)$ in the normal region $(-d<x<d)$, assuming $\hbar=1$,

$$
\left[\sigma_{z} \widehat{E}, \check{G}\right]=i \mathcal{D} \partial_{x}\left(\check{G} \partial_{x} \check{G}\right), \quad \check{G}^{2}=1, \quad \check{G}=\left(\begin{array}{cc}
\hat{g}^{R} & \widehat{G}^{K} \\
0 & \hat{g}^{A}
\end{array}\right),
$$

and the boundary conditions at the normal metal-superconductor interfaces with the resistances $R_{1}$ (right) and $R_{2}$ (left) [25],

$g_{N}\left(\check{G} \partial_{x} \check{G}\right)_{ \pm d}= \pm\left(2 R_{1,2}\right)^{-1}\left[\check{G}_{ \pm d}, \check{G}_{1,2}\right]$.

Here $\hat{g}^{R, A}$ are the retarded/advanced Green's functions, $\hat{G}^{K}=\hat{g}^{R} \hat{f}-$ $\hat{f} \hat{g}^{A}$ is the Keldysh function with the matrix distribution function $\hat{f}, \mathcal{D}$ is a diffusion constant, the kernel of the energy operator $\widehat{E}$ is $E\left(t_{1}, t_{2}\right)=i \partial_{t_{1}} \delta\left(t_{1}-t_{2}\right)$, and 'check' and 'hat' denotes $4 \times 4$ Keldysh and $2 \times 2$ Nambu matrices, respectively. All products in Eq. (1) are time convolutions, $(A B)\left(t_{1}, t_{2}\right)=\int d t A\left(t_{1}, t\right) B\left(t, t_{2}\right)$.

The equilibrium Keldysh-Green's functions $\breve{G}_{1,2}$ in the right and left reservoirs are constructed from the local-equilibrium Green's and distribution functions. In $(E, t)$-representation, $A(E, t)=$ $\int d \tau e^{i E \tau} A(t+\tau / 2, t-\tau / 2)$, they read

$\hat{g}_{1,2}=\sigma_{z} u\left(E \pm \sigma_{z} e V / 2\right)+i \exp \left( \pm i \sigma_{z} e V t\right) \sigma_{y} v(E)$,

$\hat{f}_{1,2}=\tanh \left[\left(E \pm \sigma_{z} e V / 2\right) / 2 T\right]$,

$u(E)=\frac{E}{\xi}, \quad v(E)=\frac{\Delta}{\xi}, \quad \xi^{R, A}=\sqrt{(E \pm i 0)^{2}-\Delta^{2}}$.

In Eq. (3), we use the antisymmetric gauge of the superconducting phase, $\phi_{1}=-\phi_{2}=e V t$, satisfying the Josephson relation $\phi=\phi_{1}-$ $\phi_{2}=2 e V t$.
The electric current $I(t)$ is defined as

$I(t)=\left(\pi g_{N} / 4 e\right) \operatorname{Tr} \tau_{K}\left(\check{G} \partial_{x} \check{G}\right)(t, t), \quad \tau_{K}=\sigma_{z} \tau_{x}$,

where $g_{N}$ is the conductance of the normal region per unit length, and the $\sigma$ and $\tau$ Pauli matrices operate in the Nambu and the Keldysh space, respectively.

We construct an approximate solution to Eqs. (1) and (2) by performing integration of the diffusive equation along the coordinate $x$ of the normal region, replacing $\breve{G}$ in the left-hand side with its spatially averaged value $\breve{G}$ and using the boundary condition (2),

$2 d\left[\sigma_{z} \widehat{E}, \check{G}\right]=\frac{i \mathcal{D}}{2 g_{N}}\left(\left[\check{G}_{d}, \frac{\check{G}_{1}}{R_{1}}\right]+\left[\check{G}_{-d}, \frac{\check{G}_{2}}{R_{2}}\right]\right)$.

In short junctions with opaque barriers, the resistance of which exceeds the normal resistance $R_{N}=2 d / g_{N}$ of the normal region, the function $\breve{G}$ slowly varies along the normal region [26,27], so that $\breve{G} \approx \breve{G} \approx \breve{G}_{d} \approx \breve{G}_{-d}$. This approximation leads to a simplified equation for the single quantity $\breve{G}$,

$2 d\left[\sigma_{z} \widehat{E}, \check{G}\right]=\frac{i \mathcal{D}}{2 g_{N}}\left[\check{G}, \frac{\check{G}_{1}}{R_{1}}+\frac{\check{G}_{2}}{R_{2}}\right]$.

In a similar way one can get a simplified equation for the current, taking symmetrized value of the current at the ends of the normal region and using the boundary condition (2),

$I(t)=\frac{\pi}{8 e} \operatorname{Tr} \tau_{K}\left[\check{G}, \frac{\check{G}_{1}}{R_{1}}-\frac{\check{G}_{2}}{R_{2}}\right](t, t)$.

The simplified Green's function Eq. (8) and equation for the current (9) can be written in a more compact form by introducing quantities

$\check{A}=\check{G}_{+}-i \sigma_{z} \tau \widehat{E}, \quad \check{G}_{ \pm}=\frac{1}{2}\left(r_{1} \check{G}_{1} \pm r_{2} \check{G}_{2}\right)$,

$r_{1,2}=\frac{R}{R_{1,2}}, \quad \frac{1}{R}=\frac{1}{2}\left(\frac{1}{R_{1}}+\frac{1}{R_{2}}\right), \quad \gamma=\tau \Delta=\frac{R}{R_{N}} \frac{\Delta}{E_{\mathrm{Th}}}$.

The parameter $\gamma$ introduced in Eq. (11) quantifies the effect of the electron-hole dephasing, and $\tau=E_{\mathrm{Th}}^{-1} R / R_{N}$, where the Thouless energy is defined as $E_{\mathrm{Th}}=\mathcal{D} /(2 d)^{2}$, characterizes the dwell time. In these notations, we obtain the equations

$[\check{A}, \check{G}]=0$,

$I(t)=\frac{\pi}{8 e R} \operatorname{Tr} \tau_{K}\left[\check{G}, \check{G}_{-}\right](t, t)$.

Following $[28,29]$, we write a formal solution to Eq. (12) which obeys the commutation relation in Eq. (12) and the normalization condition in Eq. (1),

$\check{G}=\check{A} / \sqrt{\check{A}^{2}}$.

A constructive form of Eq. (14) appropriate for the analysis of a nonstationary regime can be obtained by means of the integral representation [18]

$\check{G}=\frac{1}{\pi} \int_{-\infty}^{\infty} d \lambda \check{K}(\lambda), \quad \check{K}(\lambda)=(\check{A}+i \lambda)^{-1}$,

where the integral is assumed to be taken in symmetric limits which simultaneously turn to $\pm \infty$. Then Eq. (13) reads

$I(t)=\int_{-\infty}^{\infty} \frac{d \lambda}{8 e R} \operatorname{Tr} \tau_{K}\left[\check{K}(\lambda), \check{G}_{-}\right](t, t)$.

Eqs. (15) and (16) are the main technical result of the paper; they describe short asymmetric double-barrier SNS junctions for all values of parameter $\gamma$. In what follows we will apply these equations for calculation of the dc current-voltage characteristics. 
The chosen form for the solution is justified by the limit of vanishing dephasing parameter, $\gamma=0$, when Eq. (16) reduces to a known universal formula for the current through a short connector $[30,31]$. Indeed, in this case, reducing the integral in Eq. (16) to the positive axis, we have $\check{K}(\lambda)=2 \check{G}_{+}\left(\check{G}_{+}^{2}+\lambda^{2}\right)^{-1}$, and then, after simple algebra, we obtain the commutator in Eq. (16) in terms of the functions $\check{G}_{1,2}$ :

$\left[\check{K}(\lambda), \check{G}_{-}\right]=\frac{1}{2} \frac{r_{1} r_{2}\left[\check{G}_{2}, \check{G}_{1}\right]}{\lambda^{2}+\frac{1}{4}\left(r_{1}^{2}+r_{2}^{2}+r_{1} r_{2}\left\{\check{G}_{1}, \check{G}_{2}\right\}\right)}$.

Substituting Eq. (17) to Eq. (16), using the equality $r_{1}+r_{2}=2$, and introducing the transparency variable $D=r_{1} r_{2} /\left(\lambda^{2}+1\right)$, we arrive at a convolution of a non-resonant single-channel current with the transparency distribution $\rho(D)$ for an asymmetric double-barrier junction [32,33],

$$
\begin{aligned}
& I(t)=\frac{\pi}{4 e R_{T}} \int_{0}^{D_{\max }} \operatorname{Tr} \tau_{K} \frac{D \rho(D)\left[\check{G}_{2}, \check{G}_{1}\right] d D}{1+\frac{D}{4}\left(\left\{\check{G}_{1}, \check{G}_{2}\right\}-2\right)}(t, t), \\
& \rho(D)=\frac{1}{\pi D^{3 / 2} \sqrt{D_{\max }-D}}, \quad D_{\max }=r_{1} r_{2}=\frac{4 R_{1} R_{2}}{R_{T}^{2}} .
\end{aligned}
$$

where $R_{T}=R_{1}+R_{2}$ is the net resistance of the tunnel barriers.

\section{Equilibrium Josephson current and the minigap function}

Prior to the discussion of a general non-equilibrium case, it is instructive to demonstrate how to use Eqs. (15) and (16) for evaluation of the equilibrium Josephson current. In this case, the distribution function is equilibrium, $f=f_{2}=f_{1}=\tanh (E / 2 T)$, and we need to calculate only the Green's functions. In the reservoirs, they are given by $\hat{g}_{1,2}=\sigma_{z} u+i \exp \left( \pm i \sigma_{z} \phi / 2\right) \sigma_{y} v$; the solution for the Green's function $\hat{g}$ in the normal region has the form of Eq. (15) with the diagonal (retarded and advanced) component $\widehat{A}_{g}$ of the full matrix $\check{A}$ :

$\widehat{A}_{g}=\sigma_{z}(u-i \tau E)+i v\left[\sigma_{y} \cos (\phi / 2)+\sigma_{x} \kappa \sin (\phi / 2)\right]$,

$\widehat{A}_{g}^{2}=(u-i \tau E)^{2}-v^{2} \eta^{2}, \quad \eta^{2}(\phi)=\cos ^{2} \frac{\phi}{2}+\kappa^{2} \sin ^{2} \frac{\phi}{2}$,

where $\kappa=\left(R_{2}-R_{1}\right) /\left(R_{2}+R_{1}\right)$. As the result, we obtain

$\hat{g}=\widehat{A}_{g} / \sqrt{\widehat{A}_{g}^{2}}=\sigma_{z} \widetilde{u}+i \widetilde{v} \exp \left(i \sigma_{z} \Phi\right) \sigma_{y}$,

$\widetilde{u}=\frac{E}{\sqrt{E^{2}-\widetilde{\Delta}^{2}(E, \phi)}}, \quad \widetilde{v}=\frac{\widetilde{\Delta}(E, \phi)}{\sqrt{E^{2}-\widetilde{\Delta}^{2}(E, \phi)}}$,

$\widetilde{\Delta}(E, \phi)=\frac{\Delta \eta(\phi)}{1-i \gamma / v(E)}, \quad \Phi(\phi)=\arctan \left(\kappa \tan \frac{\phi}{2}\right)$.

According to Eq. (22), the minigap $\Delta_{g}(\phi)$ in the spectrum of the normal region is the solution of equation

$\Delta_{g}=\widetilde{\Delta}\left(\Delta_{g}, \phi\right)$.

As follows from Eq. (23), at $\gamma \gg 1$ and $\phi=0, \Delta_{g} \approx \Delta /(1+\gamma)$.

In strongly asymmetric junctions with essentially different resistances of the barriers, $R_{\max } \gg R_{\min }$, the transparency parameter $\gamma \approx 2 \gamma_{\text {min }}=2\left(R_{\min } / R_{N}\right)\left(\Delta / E_{\mathrm{Th}}\right)$ is determined by the smallest barrier strength. In this case, $\kappa \rightarrow 1$ and $\eta(\phi) \rightarrow 1$, therefore the minigap weakly depends on the phase difference and approaches its value at $\phi=0$, while in the symmetric case the minigap oscillates with the phase as $\Delta_{g}(0)|\cos (\phi / 2)|$. The physical explanation is as follows. In the main approximation, the stronger barrier can be considered as impenetrable wall, therefore the spectrum of the $\mathrm{N}$ region, calculated using the image method, is similar to the one for an effective SINIS junction with I referring to the more transpar- ent barrier, and $\mathrm{N}$ having doubled length (which is manifested by doubled $\gamma_{\min }$ in the estimate of $\Delta_{g}$ ). Since both $\mathrm{S}$ electrodes in such an effective junction originate from the single $S$ electrode, the effective phase difference is zero within this approximation.

Expression for the current follows from Eq. (12) in energy representation,

$I=\int_{-\infty}^{\infty} \frac{d E}{16 e R} \operatorname{Tr} \check{G}\left[\check{G}_{-}, \tau_{K}\right](E)$

Using Eqs. (21)-(23) $G^{K}=\left(g^{R}-g^{A}\right) \tanh (E / 2 T)$, we get

$I=\frac{i \sin \phi}{4 e R_{T} \eta(\phi)} \int_{-\infty}^{\infty} d E v^{R} \widetilde{v}^{R} \tanh \frac{E}{2 T}-(R \rightarrow A)$,

or in the Matsubara representation,

$I=\frac{2 \pi T}{e R_{T}} \sum_{\omega_{n}>0} \frac{1}{\sqrt{\omega_{n}^{2}+\Delta^{2}}} \frac{\Delta^{2} \sin \phi}{\sqrt{\omega_{n}^{2} q_{n}^{2}+\Delta^{2} \eta^{2}(\phi)}}$,

$q_{n}=1+\tau \sqrt{\omega_{n}^{2}+\Delta^{2}}, \quad i \omega_{n}=i \pi T(2 n+1)$.

Eq. (26) coincides with the result of a direct solution of the Usadel equation [23] and gives a general description for the Josephson current in the double-barrier junctions.

At zero temperature, Eq. (26) reduces to

$I=\frac{\Delta \sin \phi}{e R_{T}} \times \begin{cases}K\left(\sqrt{1-\kappa^{2}} \sin \frac{\phi}{2}\right), & \gamma \ll 1, \\ \frac{1}{\gamma} \ln \frac{2 \gamma}{\eta(\phi)}, & \gamma \gg 1 .\end{cases}$

where $K$ is the elliptic integral. These results have also been derived by another methods for the chaotic quantum dot in ergodic regime [34] and for a diffusive junctions with equal $[25,27]$ and asymmetric [23] barriers.

\section{Voltage biased Josephson junction}

When the voltage is applied across the junction, the proximity state in the normal region becomes nonstationary because of different time dependencies of the electrode Green's functions in Eq. (3). The periodicity of these functions allows us to expand all matrices written in the $(E, t)$-representation over the temporal harmonics, $A(E, t)=\sum_{m} A(E, m) e^{-i m e V t}$.

In this representation, the time averaged (dc) current $I=\overline{I(t)}$ reads

$I=\int_{-\infty}^{\infty} \int_{-\infty}^{\infty} \frac{d \lambda d E}{16 \pi e R} \sum_{m} \operatorname{Tr} \check{K}(\lambda, E, m)\left[\check{G}_{-}(E,-m), \tau_{K}\right]$.

Due to the fact that the local-equilibrium Green's functions in the electrodes ( 3 ) contain only three harmonics, $m=0$ and \pm 1 , the current consists of only three respective terms. By the same reason, equation for the matrix $\check{K}$ in Eq. (15), $(\check{A}+i \lambda) \breve{K}(\lambda)=1$, takes the form of the three-term recurrency,

$$
\begin{aligned}
& {\left[\check{G}_{+}\left(E_{m}, 0\right)-i \sigma_{z} \tau E_{m}+i \lambda\right] \check{K}_{m}+\check{G}_{+}\left(E_{m-1 / 2}, 1\right) \check{K}_{m-1}} \\
& \quad+\check{G}_{+}\left(E_{m+1 / 2},-1\right) \check{K}_{m+1}=\delta_{m, 0},
\end{aligned}
$$

where $K_{m}(E)=K(E+m e V / 2, m)$ and $E_{k}=E+k e V$.

In order to make the analysis of Eqs. (28) and (29) more tractable, we perform in this section some manipulations with the matrices $\breve{K}_{m}$ and $\breve{G}_{ \pm}$, in order to reveal the symmetries and simplify the structure of the recurrence equation.

We start by introducing specific notations for the real-valued components of the BCS Green's functions (5),

$N=\operatorname{Re} u^{R}, \quad M=\operatorname{Re} v^{R}, \quad \bar{N}=\operatorname{Im} u^{R}, \quad \bar{M}=\operatorname{Im} v^{R}$,

$(N, M)(E) \propto \theta\left(E^{2}-\Delta^{2}\right), \quad(\bar{N}, \bar{M})(E) \propto \theta\left(\Delta^{2}-E^{2}\right)$, 
where $\theta(x)$ is the Heaviside step function, $N(E)$ is the BCS density of states, and write the functions $\breve{G}_{ \pm}$explicitly,

$\check{G}_{ \pm}(E, m)=\sigma_{z} \delta_{m, 0} \sum_{\sigma= \pm} \hat{t}_{\sigma}^{ \pm} G_{0}^{+}\left(E_{\sigma}\right)+i \sigma_{y} \hat{t}_{m}^{ \pm} G_{1}^{+}(E)$.

Here we use the following abbreviations

$G_{0}^{+}\left(E_{\sigma}\right)=\frac{1}{2}\left(i \bar{N}_{\sigma}+N_{\sigma} F_{\sigma}\right), \quad G_{1}^{+}(E)=\frac{1}{2}(i \bar{M}+M F)$,

$\hat{t}_{\sigma}^{ \pm} \equiv r_{1} \hat{p}_{\sigma} \pm r_{2} \hat{p}_{-\sigma}, \quad F=\tau_{z}+2 f \tau_{+}, \quad \sigma= \pm$,

where $\quad E_{ \pm}=E \pm e V / 2, A_{ \pm}=A\left(E_{ \pm}\right), \tau_{+}=(1 / 2)\left(\tau_{x}+i \tau_{y}\right), \hat{p}_{\sigma}=(1+$ $\left.\sigma \sigma_{z}\right) / 2$ are projectors in the Nambu space, and the tensor products of the Nambu matrices $\hat{t}$ and $2 \times 2$ Keldysh matrices $G_{0,1}^{+}$are assumed in Eq. (31). For brevity, here and in the following we will avoid any special notations for such matrices in the Keldysh space, keeping 'check' for the $4 \times 4$ matrices and 'hat' for the $2 \times 2 \mathrm{Nambu}$ matrices.

Eq. (29) can be presented in a more compact form,

$\left(\check{Q}_{m}-i \tau E_{m}+i \sigma_{z} \lambda\right) \check{K}_{m}+\sigma_{x}\left(\check{q}_{m-1} \check{K}_{m-1}+\check{q}_{m}^{\prime} \check{K}_{m+1}\right)=\sigma_{z} \delta_{m, 0}$,

after multiplying Eq. (29) by $\sigma_{z}$ and introducing notations

$\check{Q}_{m}(E)=\hat{t}_{+}^{+} H_{m}+\hat{t}_{-}^{+} H_{m-1}, \quad H_{m}=G_{0}^{+}\left(E_{m+1 / 2}\right)$,

$\check{q}_{m}=\hat{t}_{+}^{+} G_{m}, \quad \check{q}_{m}^{\prime}=\hat{t}_{-}^{+} G_{m}, \quad G_{m}=G_{1}^{+}\left(E_{m+1 / 2}\right)$.

According to the definition of $\hat{t}_{\sigma}^{+}$in Eq. (33), the prime sign in Eq. (36) means the change $\sigma_{z} \rightarrow-\sigma_{z}$, or $\hat{p}_{+} \leftrightarrow \hat{p}_{-}$, or $r_{1} \leftrightarrow r_{2}$.

Now we show that the $4 \times 4$ matrix recurrence Eq. (34) can be simplified and written in terms of the $2 \times 2$ matrices. Let us assume the ansatz

$\check{K}_{m}= \begin{cases}\sigma_{x} \check{P}_{m} \sigma_{x} \check{P}_{m-1} \ldots \sigma_{x} \check{P}_{1} \breve{K}_{0}, & m>0, \\ \sigma_{x} \check{P}_{m} \sigma_{x} \check{P}_{m+1} \ldots \sigma_{x} \check{P}_{-1} \check{K}_{0}, & m<0,\end{cases}$

which gives the recurrences for $\check{P}_{m}$, and also the expression for $K_{0}$ on the form,

$\check{P}_{m}=- \begin{cases}\left(\check{Q}_{m}^{\prime}-i \tau E-i \sigma_{z} \lambda+\check{q}_{m}^{\prime} \check{P}_{m+1}^{\prime}\right)^{-1} \check{q}_{m-1}, & m>0, \\ \left(\check{Q}_{m}^{\prime}-i \tau E-i \sigma_{z} \lambda+\check{q}_{m-1} \check{P}_{m-1}^{\prime}\right)^{-1} \check{q}_{m}^{\prime}, & m<0,\end{cases}$

$\check{K}_{0}=\sigma_{z}\left(\check{Q}_{0}-i \tau E+i \sigma_{z} \lambda+\check{q}_{0} \check{P}_{1}+\check{q}_{-1}^{\prime} \check{P}_{-1}\right)^{-1}$

According to Eqs. (33), (35), and (36), all quantities in Eqs. (38) and (39) are diagonal in the Nambu space, and therefore these $4 \times 4$ matrix relations split into a pair of $2 \times 2$ separate relations for the diagonal triangle Keldysh blocks $P_{m}$ and $K_{0}$ of the full $4 \times 4$ matrices $\check{P}_{m}$ and $\breve{K}_{0}$, respectively. These blocks differ one from another by change of the sign of $\lambda$ (since $\lambda$ enters only through the product $\sigma_{z} \lambda$ ) and by replacing $r_{1} \leftrightarrow r_{2}$, in accord with the structure of $\hat{t}_{\sigma}$.

Consider, for example, the upper block in the recurrences Eq.

(38) for $m>0$. Denote $P_{m}^{\prime}=P_{m}$ for $m=2 k$, then

$P_{1}=-\left(r_{2} H_{1}+r_{1} H_{0}-i \tau E-i \lambda+r_{2} G_{1} P_{2}\right)^{-1} r_{1} G_{0}$,

$P_{2}=-\left(r_{1} H_{2}+r_{2} H_{1}-i \tau E+i \lambda+r_{1} G_{2} P_{3}\right)^{-1} r_{2} G_{1}$,

We see that the recurrence coefficients with even index $m$ have the prefactor $r_{1}$, while the coefficients with odd $m$ are multiplied by $r_{2}$. Thus, introducing the notations

$\rho_{m}=\left\{\begin{array}{ll}r_{1}, & m=2 k, \\ r_{2}, & m=2 k+1,\end{array} \quad g_{m}=\rho_{m} G_{m}\right.$,

$h_{m}=\rho_{m} H_{m}+\rho_{m-1} H_{m-1}-i \tau E_{m}+i(-1)^{m} \lambda$,
$\Pi_{m}= \begin{cases}g_{m-1} P_{m}, & m>0, \\ g_{m} P_{m}, & m<0,\end{cases}$

and using a similar procedure for $m<0$, we finally arrive at the equation for $2 \times 2$ matrices $\Pi_{m}$,

$\Pi_{m}=- \begin{cases}g_{m-1}\left(h_{m}+\Pi_{m+1}\right)^{-1} g_{m-1}, & m>0, \\ g_{m}\left(h_{m}+\Pi_{m-1}\right)^{-1} g_{m}, & m<0,\end{cases}$

$K_{0}=\left(h_{0}+\Pi_{1}+\Pi_{-1}\right)^{-1}$.

Similar equation is valid for the lower Nambu block of the full Keldysh matrices with the change $\Lambda \rightarrow-\Lambda$, where we introduce the notation $\Lambda$ for the set $\left(\lambda, r_{1}, r_{2}\right)$ and $-\Lambda$ for $\left(-\lambda, r_{2}, r_{1}\right)$.

Thus, in these terms, the three matrices $\check{K}(E, m), m=0, \pm 1$, only relevant in the dc current in Eq. (28), take the form,

$\check{K}(E, 0)=\check{K}_{0}(E)=\hat{p}_{+} K_{0}(E, \Lambda)-\hat{p}_{-} K_{0}(E,-\Lambda)$,

$\check{K}(E, \pm 1)=\check{K}_{ \pm 1}\left(E_{\mp}\right)=\sigma_{x}\left(\check{P}_{ \pm 1} \check{K}_{0}\right)\left(E_{\mp}\right)$,

$\check{P}_{m}(E)=\hat{p}_{+} P_{m}(E, \Lambda)+\hat{p}_{-} P_{m}(E,-\Lambda)$.

\section{Current-voltage characteristics}

As noted in the previous Section, the current spectral density in Eq. (28) can be written as the sum of three terms,

$\operatorname{Tr} \sum_{m=0, \pm 1} \check{K}(E, m)\left[\check{G}_{-}(E,-m), \tau_{K}\right]=j_{0}+j_{1}+j_{-1}$,

$j_{0}=\operatorname{Tr} \check{K}_{0}(E) \sum_{\sigma= \pm} \hat{t}_{\sigma}^{-} G_{0}^{-}\left(E_{\sigma}\right)$,

$j_{ \pm 1}=-\operatorname{Tr} \check{K}(E, \pm 1) \sigma_{x} \hat{t}_{\mp}^{-} G_{1}^{-}(E)$

$=-\operatorname{Tr} \hat{t}_{ \pm}^{-} G_{1}^{-}\left(E_{ \pm}\right)\left(\check{P}_{ \pm 1} \check{K}_{0}\right)(E)$,

$G_{0}^{-}\left(E_{\sigma}\right)=\tau_{z} N_{\sigma} f_{\sigma}+i \tau_{y} N_{\sigma}, \quad G_{1}^{-}(E)=i \tau_{x} \bar{M}+M f$.

In Eq. (48) we used Eqs. (44) and (37), then shifted the energy by $\pm e V / 2$ which holds the result of integration over energy in Eq. (28) unchanged. A direct calculation of the partial current density in Eq. (47) yields

$j_{0}=r_{1} N_{+}\left(2 K_{0}^{z} f_{+}-K_{0}^{+}\right)-r_{2} N_{-}\left(2 K_{0}^{z} f_{-}-K_{0}^{+}\right)+(\Lambda \rightarrow-\Lambda)$.

Here and in the following, the upper indices $z$ and + denote $\tau_{z^{-}}$and $\tau_{+}$-components of the Keldysh matrices, respectively. We note that the change of sign of $\lambda$ in the last term to this equation plays no role because of integration over $\lambda$ in Eq. (28); moreover, due to the symmetries of the spectral functions in Eq. (49) with respect to $E \rightarrow-E$ (see Appendix A), this term simply doubles the contribution of the upper line into the full current.

Analysis of the contributions Eq. (48) of the first harmonics performed in the A [see Eqs. (A.7), (A.8), (A.9), (A.10), (A.11)] shows that all terms with unity components of the matrices $K_{0}$ and $\Pi$ cancel each other after integration over $E$ and $\lambda$ in Eq. (28). As the result, we finally arrive at the following simplified expression for the dc current,

$$
\begin{aligned}
I=\int_{-\infty}^{\infty} & \int_{-\infty}^{\infty} \frac{d E d \lambda}{16 \pi e R}\left(j_{0}+j_{1}+j_{-1}\right) \\
j_{1}+j_{-1}= & \theta\left(\Delta^{2}-E_{-}^{2}\right)\left(K^{+} \Pi_{-1}^{z}-K^{z} \Pi_{-1}^{+}\right) \\
& \quad-\theta\left(\Delta^{2}-E_{+}^{2}\right)\left(K^{+} \Pi_{1}^{z}-K^{z} \Pi_{1}^{+}\right)+(\Lambda \rightarrow-\Lambda)
\end{aligned}
$$

with $j_{0}$ given by Eq. (49). As mentioned in comments to Eq. (49), the change $\Lambda \rightarrow-\Lambda$, due to integration over $\lambda$, can be reduced to the permutation $r_{1} \leftrightarrow r_{2}$. 


\subsection{Numerical results}

Numerical computation of current-voltage characteristics (IVCs) was done using Eqs. (50) and (51) with the function $K_{0}$ defined in (42) and the solution $\Pi_{ \pm 1}$ of the recurrence (41). In this paper we focus on the case, opposite to the one studied earlier [18] of a large difference between the barrier transparencies, say, $\gamma_{1} \gg \gamma_{2}$, where $\gamma_{1,2}=\left(R_{1,2} / R_{N}\right)\left(\Delta / E_{\mathrm{Th}}\right), \gamma^{-1}=(1 / 2)\left(\gamma_{1}^{-1}+\gamma_{2}^{-1}\right)$. In this case, the strongest barrier plays the role of a tunnel probe for the junction spectrum formed basically by the weakest barrier, as was explained in comments to Eq. (24). On this account, we keep the relation $\gamma_{1}=10 \gamma_{2}$, or, equivalently, $R_{1}=10 R_{2}$ while calculating the IVCs at different $\gamma_{1,2}$.

In Fig. 1, the results of numerical computation of the IVCs are shown for several sets of $\gamma_{1,2}$. As one can see in Fig. 1(a), the excess current at large voltage is very small even at rather small $\gamma$, and rapidly becomes negative, i.e., transforms to the deficit current, as long as $\gamma$ increases. This is due to the strong asymmetry of the junction assumed in our calculations, which confines the distribution of transparency coefficients within the small enough interval, $0<D<D_{\max } \approx 0.4$. Such a suppression of the excess current is similar to the case of a junction with a single strong barrier.

For transparent barriers, $\gamma_{2}=0.1$, the IVC is close, as expected, to the result of averaging of the current through a single-mode point contact over the transparency distribution in a normal double-barrier structure, see Eqs. (18) and (19). In this case, the steps in the IVC scale as $\left(D_{\max } / 2\right)^{-2 \Delta / e V}$; similar scaling has been found for the tunnel junction with fixed transparency $D$ within the framework of multiparticle tunneling theory [35-37] and MAR theory [15-17]. The subharmonic features [shown by downward arrows above the curve 1 in Fig. 1(b)] are well fitted with the "combination" subharmonics of the quantity $\Delta+\Delta_{g}$, although they are quite close to the standard subharmonics of the bulk energy gap $2 \Delta$. The
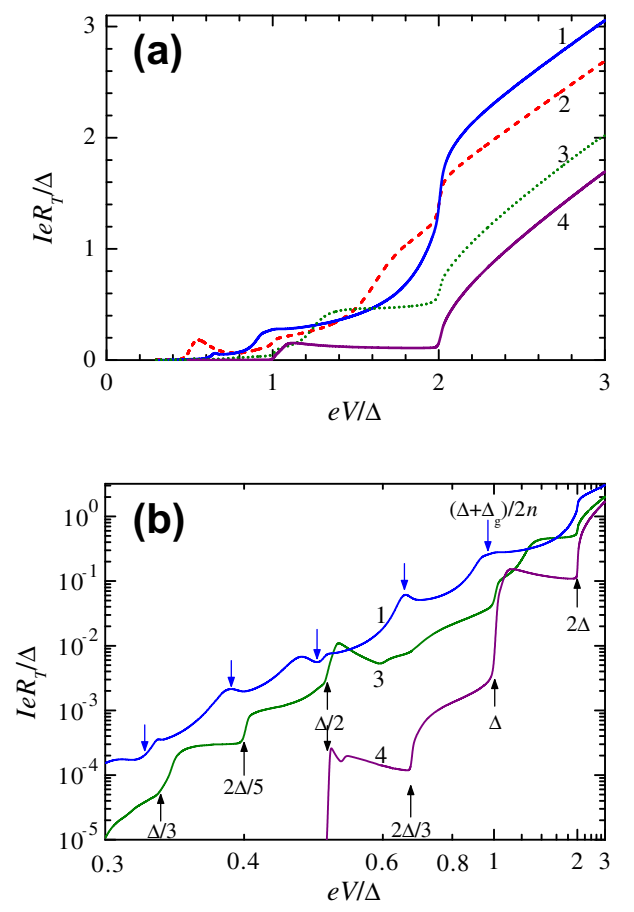

Fig. 1. dc Current vs applied voltage at $T=0$ for different transparency parameters: $\gamma_{2}=0.1, \gamma_{1}=1, \gamma=0.18, \Delta_{g}=0.94 \Delta \quad$ (curve 1); $\gamma_{2}=0.5, \gamma_{1}=5, \gamma=0.91$, $\Delta_{g}=0.57 \Delta$ (curve 2); $\gamma_{2}=2, \gamma_{1}=20, \gamma=3.64, \Delta_{g}=0.22 \Delta$ (curve 3 ); $\gamma_{2}=$ $10, \gamma_{1}=100, \gamma=18.2, \Delta_{g}=0.052 \Delta$ (curve 4). (a) - current vs voltage in linear scale, (b) - current in logarithmic scale vs voltage in reciprocal scale. Downward arrows above the curve 1 indicate subharmonics of $\Delta+\Delta_{g}$ for small values of $\gamma$. latter is explained by the fact that for transparent barriers, the minigap $\Delta_{g}$ approaches $\Delta$.

With increasing barrier strengths, $\gamma_{2}=2-10$, the junction enters the regime of strong dephasing, $\gamma \gg 1$. In this case, the role of the effective tunneling parameter is played by $\gamma^{-1}$, as it was noted in [18], and, correspondingly, the IVC steps scale as $\gamma^{-24 / e V}$. This conclusion is confirmed by asymptotic analysis of multiparticle currents presented in next subsection. The gap subharmonics correspond to the current onsets [see Fig. 1(b)], i.e., to maxima of the differential conductance $d I / d V$. Such maxima are shown in Fig. 2, together with clearly pronounced peaks at $\mathrm{eV}=\Delta+\Delta_{g}$. The latter peaks are explained by enhanced transmissivity of MAR chains containing links between the edges of the minigap and the bulk gap, where the density of state is enhanced [38]. This effect is analogous to the one in single channel resonant junctions $[39,40]$, where additional peaks appear on IVC at voltages related to positions of geometric or Andreev resonances in equilibrium.

Interestingly, similar splitting of the conductance peak near $e V=\Delta$ has been found in [41] for S-chaotic dot-S junction with the minigap of the order of small Thouless energy $E_{\mathrm{Th}}<\Delta$, which corresponds to the long junction regime. The conductance peak at $\Delta+\Delta_{g}$ has also been noted for an SNS junction with transparent interfaces [42] and for a point contact between massive SN sandwiches [14]. The physics in the latter case is similar to the situation in asymmetric double-barrier junction considered in this paper: the minigap is basically formed by the proximity effect in wellcoupled $\mathrm{S}$ and $\mathrm{N}$ regions, while the weak link, i.e., the point contact (in our case - the strongest barrier) plays a role of a probe, which weakly affects the spectrum but detects its features in the IVC. Thus, the appearance of this specific feature can be considered as a rather general phenomenon, which has also been observed in experiments [43-45]. We note that the strong asymmetry of the junction provides the most favorable conditions for this effect: as noted above, in this case the minigap $\Delta_{g}(\phi)$ insignificantly depends on the superconducting phase difference $\phi(t)$ and therefore holds nearly constant value $\Delta_{g}(0)$.

At very large $\gamma$ [curve 4 in Fig. 1(a)], the minigap is small, $\Delta_{g} \approx 0.05$, and therefore the splitting of the SGS at $\Delta+\Delta_{g}$ remains visible only in the differential conductance while the IVC features almost exactly correspond to the subharmonics of the superconducting gap. In this case, the presence of the minigap manifests itself in the IVC as anomalous enhancement of the magnitude of the dc current just above the even gap subharmonics. This effect is due to the enhanced density of states in the vicinity of the minigap which increases the transmissivity of the MAR trajectories having even number of steps and therefore simultaneously touching the superconducting gap edges and the small minigap region in the middle of the bulk gap. This resonance effect becomes more

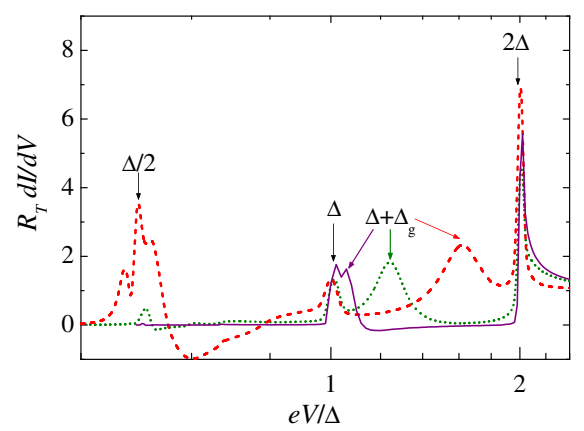

Fig. 2. Differential conductance vs voltage at $T=0: \gamma_{2}=0.5, \gamma_{1}=5, \Delta_{g}=0.57 \Delta$ (dashed curve); $\gamma_{2}=2, \quad \gamma_{1}=20, \Delta_{g}=0.22 \Delta \quad$ (dotted curve); $\gamma_{2}=10$, $\gamma_{1}=100, \Delta_{g}=0.052 \Delta$ (solid curve). The subharmonics of the energy gap $2 \Delta$ and the peaks at $e V=\Delta+\Delta_{g}$ are shown by arrows. 
pronounced for higher subharmonics and leads to the appearance of the IVC portions with negative differential conductance [see curves 3 and 4 in Fig. 1(b)].

\subsection{Some analytical results}

As it was mentioned, in junctions with a small dephasing parameter $\gamma \ll 1$, the problem reduces to the point contact limit [14], and eventually to the single channel problem, which has been extensively studied [15-17]. Here we present some analytical results for the opposite limit of large dephasing $\gamma \gg 1$. In this case, it is possible to express analytically the full dc current as a sum of contributions of $n$-particle tunneling processes, similar to the single channel theory [15]. Solutions of the recurrence (41) for the quantities $\Pi_{m}$, which determine all functions necessary for the calculation of the dc current in Eq. (50), can be presented as perturbative expansion series over the powers of $\gamma^{-1}$. Physically, these expansions reflect the nature of the net current as a sum of $n$-particle tunnel currents; each of them exists at $\mathrm{eV}>2 \Delta / n$ and scales as $\gamma^{1-n}$ with respect to the single-particle current. The latter fact allows us to consider the $n$-particle current $I^{(n)}$ only within its actual voltage region $2 \Delta / n<e V<2 \Delta /(n-1)$; at larger voltages, the $(n-1)$-particle current dominates. This simplifies further calculations and enables us to present the net current in the form

$I=\sum_{n=1}^{\infty} \chi_{n}(V) I^{(n)}$

$\chi_{n}(V)= \begin{cases}1, & 2 \Delta / n<e V<2 \Delta /(n-1) \\ 0 & \text { otherwise }\end{cases}$

Estimation shows that the $m$ th term in the perturbative expansion for $\Pi_{m}$ contributes to the $(m+1)$-particle current; thus, it suffices to consider them only at $\mathrm{eV}<2 \Delta / \mathrm{m}$, which greatly simplifies the structure of the series.

We refer the reader to the $B$ for the details of the evaluation of the partial currents, which are rather cumbersome due to the junction asymmetry. According to Eq. (B.2), the n-particle current consists of $n$ equal contributions of MAR chains with $n$ steps. Each chain starts at the energy $E<-\Delta$ and finishes at $E>\Delta$, thus transferring the quasiparticles to the extended states above the energy gap. The intermediate points in this chain correspond to the energies inside the gap at which the Andreev reflections take place. Here we present only final results for the first three partial currents and the excess current.

The single-particle current exists at $e V>2 \Delta$ and can be rather straightforwardly evaluated for arbitrary temperatures,

$$
\begin{aligned}
I^{(1)}= & \int_{\Delta-e V / 2}^{-\Delta+e V / 2} \frac{d E}{2 e} \frac{N_{+} N_{-}\left(f_{+}-f_{-}\right)}{R_{1} N_{+}+R_{2} N_{-}} \\
& +\int_{\Delta+e V / 2}^{\infty} \frac{d E}{2 e} N_{+} N_{-} \times\left(f_{+}-f_{-}\right)\left(\frac{1}{R_{1} N_{+}+R_{2} N_{-}}+\frac{1}{R_{2} N_{+}+R_{1} N_{-}}\right)
\end{aligned}
$$

(we remind that the subscripts \pm denote the energy shift by $\pm e V / 2$ ).

The spectral density of the two-particle current calculated at $\Delta<e V<2 \Delta$ has a resonant form, with a sharp peak at zero energy. If the applied voltage is not very close to the threshold $\Delta / e$ of the two-particle current, the corresponding integral over energy can be calculated in the resonant approximation. For simplicity, we present only the result for $T \ll \Delta$,

$I^{(2)}=\frac{\pi \Delta N(e V)}{2 e R_{T} \gamma_{1} \gamma_{2}} \sum_{i=1,2} \frac{\gamma_{i}}{\sqrt{1+r_{i}^{2} N^{2}(e V)}}$.

The three-particle current within the main approximation in $\gamma^{-1}$ at $T \ll \Delta$ reads

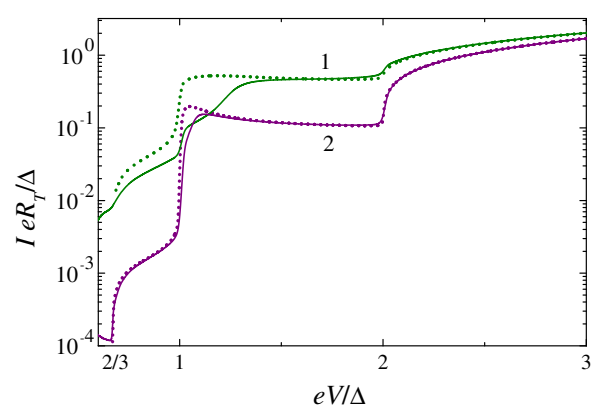

Fig. 3. Comparison of the results of numerical calculation (solid lines) and analytical approximation (dotted lines) for the contribution of the 1-, 2-, and 3-particle currents to the net dc current: $\gamma_{2}=2, \gamma_{1}=20$ (curves 1 ); $\gamma_{2}=10, \gamma_{1}=$ 100 (curves 2).

$I^{(3)}=\frac{3}{4 e \gamma_{1} \gamma_{2}} \int_{\Delta-3 e V / 2}^{-\Delta+3 e V / 2} \frac{N_{3 / 2} S_{+} S_{-} N_{-3 / 2} d E}{R_{1} S_{-} N_{-3 / 2} E_{+}^{2}+R_{2} S_{+} N_{3 / 2} E_{-}^{2}}$,

where $N_{ \pm 3 / 2}=N(E \pm 3 e V / 2)$ and $s(E)=\bar{M}^{2}(E) / 4$. The numerator in this equation clearly illustrates the structure of the relevant MAR chain: it starts below the superconducting gap at the energy $E-3 e V / 2$, then the particle experiences Andreev reflections inside the gap at the points $E \pm e V / 2$, and finishes above the gap, at the energy $E+3 e V / 2$. Fig. 3 demonstrates a rather good agreement between our purely numerical and analytical results for the junction with opaque barriers, i.e., at large enough barrier strength $\gamma$.

According to the definition, the excess current $I^{\text {exc }}$ is the voltageindependent term in asymptotic expression for the dc current $I=V / R_{T}+I^{e x c}+O(\Delta / e V)$ at $e V \gg \Delta$. It is contributed by the single-particle current and the two-particle (Andreev) current, and can be evaluated for arbitrary $\gamma$, see $C$, where we restrict our consideration to $T=0$. In the limit $\gamma \gg 1, I^{e x c}$ appears to be negative (deficit current), as one may expect for an opaque junction,

$I^{e x c}=-\frac{\Delta}{e R_{T}}\left[1+\frac{1-a^{2}}{2 a^{3 / 2}}\left(\frac{1}{2} \ln \frac{1+\sqrt{a}}{1-\sqrt{a}}-\arctan \sqrt{a}\right)\right]$,

$\gamma \gg 1, \quad a^{2} \equiv 1-r_{1} r_{2}$

$I^{e x c}=-\frac{\Delta}{e R_{T}} \begin{cases}4 / 3, & r_{1,2}=1(\text { symmetric junction }) ; \\ 1, & r_{1} r_{2} \ll 1 \text { (strong asymmetry). }\end{cases}$

For rather transparent interfaces, $\gamma \ll 1$, the excess current can be expressed through the convolution of its value for a single ballistic channel [46] with the transparency distribution (19),

$I^{\text {exc }}=\frac{\Delta}{e R_{T}} \int_{0}^{D_{\max }} \frac{D^{2}}{\mathcal{R}}\left[1-\frac{D^{2}}{2 \sqrt{\mathcal{R}(\mathcal{R}+1)}} \ln \frac{1+\sqrt{\mathcal{R}}}{1-\sqrt{\mathcal{R}}}\right] \times \rho(D) d D$,

$\mathcal{R}=1-D$.

\section{Conclusion}

In conclusion, we have presented theoretical investigation of the current-voltage characteristics in diffusive asymmetric SINIS Josephson junctions with a short but finite length and different transparencies of SIN interfaces. Our theory is relevant for current transport in Josephson devices with multichannel semiconducting nanowires and multilayered planar metallic junctions. We have shown that the coherent multiple Andreev reflection theory can be efficiently developed and analyzed in detail, both numerically and analytically, for the whole range of the interface transparencies and arbitrary asymmetry. 
We focused on the limit of resistive interfaces, when the dwell time of the quasiparticles in the normal region becomes large, and the length dependence of the transport characteristics becomes essential. Furthermore, we found that in the case of significant asymmetry of the interface resistances, the subgap current structures contain pronounced combination subharmonics of the bulk energy gap and the proximity minigap, $\Delta+\Delta_{g}$, in addition to the conventional subharmonics of the bulk energy gap $2 \Delta$. The effect of the proximity minigap on the subgap current structures was found in a number of numerical studies of various kinds of disordered SNS junctions, and also observed in experiments. We argue, based on the detailed analytical study, that this novel subgap structure is a robust feature and a general property of diffusive SNS junctions.

In the limit of rather transparent interfaces, our theory recovers a known formula for a short mesoscopic connector - a convolution of the current through a single-channel point contact with the transparency distribution for an asymmetric double-barrier potential.

\section{Appendix A. Symmetries}

In this section we discuss distinctive symmetries of the matrices $\Pi$ and $K_{0}$, which allow us to simplify the expression for the dc current. For simplicity, we first consider the case of equal barriers, $r_{1}=r_{2}=1$. Beginning from the analysis of the symmetries of the matrix $h_{m}$, we will assume in this section all spectral and distribution functions to be dependent on the energy $E+e V / 2$, i.e., $N_{m} \equiv N[E+e V(m+1 / 2)]$. This allows us to write down the expansion of the matrix $h_{m}$ over the Pauli matrices in the Keldysh space in the following form

$$
\begin{aligned}
h_{m}= & i\left[\bar{N}_{m}+\bar{N}_{m-1}-\tau E_{m}+(-1)^{m} \lambda\right]+\tau_{z}\left(N_{m}+N_{m-1}\right) \\
& +2 \tau_{+}\left(N_{m} f_{m}+N_{m-1} f_{m-1}\right) .
\end{aligned}
$$

Due to such indexing, the symmetry relations for the spectral and distribution functions with respect to the change $E \rightarrow-E$ read as

$N_{m}(-E)=N_{m^{\prime}}(E), \quad \bar{N}_{m}(-E)=-\bar{N}_{m^{\prime}}(E)$,

$M_{m}(-E)=-M_{m^{\prime}}(E), \quad \bar{M}_{m}(-E)=\bar{M}_{m^{\prime}}(E)$,

$f_{m}(-E)=-f_{m^{\prime}}(E), \quad m^{\prime}=-m-1$.

By applying the transformation $(E, \lambda) \rightarrow(-E,-\lambda)$ to the function $h_{m}$ in Eq. (A.1) and using Eqs. (A.2), we obtain the following relations for its $1-, \tau_{z^{-}}$and $\tau_{+}$-components denoted by corresponding upper indices,

$h_{m}^{1,+}(-E,-\lambda)=-h_{-m}^{1,+}(E, \lambda)$,

$h_{m}^{z}(-E,-\lambda)=h_{-m}^{z}(E, \lambda)$.

In what follows, the Keldysh matrices with such symmetry properties will be referred to as $h$-matrices. It is easy to see that the inverse $h$-matrix is a $h$-matrix too.

Now we will prove that the matrix

$\pi_{m}(E, \lambda)= \begin{cases}g_{m-1} h_{m}^{-1} g_{m-1}, & m>0, \\ g_{m} h_{m}^{-1} g_{m}, & m<0,\end{cases}$

belongs to the class of $h$-matrices. By using the definitions of the functions $g_{m}$ and $G_{m}$ in Eqs. (36) and (32), we get $g_{m}=(1 / 2)$ $\left(i \bar{M}_{m}+\tau_{z} M_{m}\right)+f_{m} M_{m} \tau_{+}$. Denoting for brevity the $h$-matrix $h_{m}^{-1}$ as $\bar{h}_{m}$, we obtain at $m>0$

$$
\begin{aligned}
\pi_{m}(E, \lambda) & =g_{m-1} \bar{h}_{m} g_{m-1} \\
& =-(1 / 4) \bar{M}_{m-1}^{2} \bar{h}_{m}+(1 / 4) M_{m-1}^{2}\left[\bar{h}_{m}^{1}+\tau_{z} \bar{h}_{m}^{z}+\tau_{+}\left(4 f_{m-1} \bar{h}_{m}^{z}-\bar{h}_{m}^{+}\right)\right] .
\end{aligned}
$$

Replacing $(E, \lambda) \rightarrow(-E,-\lambda)$ in Eq. (A.5), using Eqs. (A.2), and comparing the result with the expression for $\pi_{-m}(E, \lambda)$ with negative indices,

$$
\begin{aligned}
\pi_{-m}(E, \lambda)= & g_{-m} \bar{h}_{-m} g_{-m} \\
= & -(1 / 4) \bar{M}_{-m}^{2} \bar{h}_{-m}+(1 / 4) M_{-m}^{2}\left[\bar{h}_{-m}^{1}+\tau_{z} \bar{h}_{-m}^{z}\right. \\
& \left.+\tau_{+}\left(4 f_{-m} \bar{h}_{-m}^{z}-\bar{h}_{-m}^{+}\right)\right],
\end{aligned}
$$

we see that the components of the matrix $\pi_{m}$ indeed satisfy Eqs. (A.3). Comparison of the definitions of the matrices $\Pi_{m}$ and $\pi_{m}$, and the fact that the sum of $h$-matrices is the $h$-matrix too, allows us to conclude that $\Pi_{m}$ is the $h$-matrix.

By using the expression Eq. (42) for the matrix $K_{0}$ through the matrices $\Pi_{ \pm 1}$ and the symmetry relations Eqs. (A.2) and (A.3), we see that $K_{0}$ is the $h$-matrix with zero index, i.e.,

$K_{0}^{1,+}(-E,-\lambda)=-K_{0}^{1,+}(E, \lambda), \quad K_{0}^{z}(-E,-\lambda)=K_{0}^{z}(E, \lambda)$.

A generalization for the case of different barriers is rather obvious: since the parity of indices of the spectral and distribution functions changes after the transformation $E \rightarrow-E$ [see Eqs. (A.2)], the symmetry relations Eqs. (A.3) for the $h$-matrices must additionally involve the change $r_{1} \leftrightarrow r_{2}$, in accordance with the definition Eq. (40a) of the function $\rho_{m}$. In our notations, this is reduced to the substitution $\lambda \rightarrow \Lambda$ in Eqs. (A.3).

Now we consider the contribution of the first harmonics to the current density Eq. (46). Using Eqs. (48) and (43)-(45), we obtain

$j_{1}=-\operatorname{Tr} \hat{t}_{+}^{-} G_{1}^{-}\left(E_{+}\right) \check{P}_{1} \check{K}_{0}=2 \operatorname{Tr}_{\tau}\left[U_{+} \Pi_{1} K_{0}+(\Lambda \rightarrow-\Lambda)\right]$,

$j_{-1}=-2 \operatorname{Tr}_{\tau}\left[U_{-} \Pi_{-1} K_{0}+(\Lambda \rightarrow-\Lambda)\right]$

$U_{ \pm}=\frac{G_{1}^{-}\left(E_{ \pm}\right) G_{1}^{+}\left(E_{ \pm}\right)}{\left[G_{1}^{+}\left(E_{ \pm}\right)\right]^{2}} \rightarrow \frac{\tau_{x} \bar{M}_{ \pm}^{2}-\tau_{z} M_{ \pm}^{2} f_{ \pm}}{M_{ \pm}^{2}-\bar{M}_{ \pm}^{2}}$.

Here we used the fact that $\left(G_{1}^{+}\right)^{2}$ is proportional to unity matrix and omitted the terms with the matrix $\tau_{+}$, the trace of the product of which with any triangle Keldysh matrix is zero.

It can be proved that all terms in the current spectral densities $j_{ \pm 1}$, which contain unity matrix components, give no contribution to the full dc current. Indeed, let us first consider the contributions of terms, proportional to $\bar{M}^{2}$ :

$$
\begin{aligned}
j_{1}^{\bar{M}} & =-2 \theta\left(\Delta^{2}-E_{+}^{2}\right) \operatorname{Tr}_{\tau} \tau_{x}\left[\Pi_{1} K_{0}+(\Lambda \rightarrow-\Lambda)\right] \\
& =-2 \theta\left(\Delta^{2}-E_{+}^{2}\right)\left[\left(\Pi_{1}^{1}+\Pi_{1}^{z}\right) K_{0}^{+}+\Pi_{1}^{+}\left(K_{0}^{1}-K_{0}^{z}\right)+(\Lambda \rightarrow-\Lambda)\right],
\end{aligned}
$$

$$
\begin{aligned}
j_{-1}^{\bar{M}} & =2 \theta\left(\Delta^{2}-E_{-}^{2}\right) \operatorname{Tr}_{\tau} \tau_{x}\left[\Pi_{-1} K_{0}+(\Lambda \rightarrow-\Lambda)\right] \\
& =2 \theta\left(\Delta^{2}-E_{-}^{2}\right)\left[\left(\Pi_{-1}^{1}+\Pi_{-1}^{z}\right) K_{0}^{+}+\Pi_{-1}^{+}\left(K_{0}^{1}-K_{0}^{z}\right)+(\Lambda \rightarrow-\Lambda)\right] .
\end{aligned}
$$

By using the symmetries (A.3) of the $h$-matrices $\Pi$ and $K_{0}$, the term $\theta\left(\Delta^{2}-E_{-}^{2}\right)\left(\Pi_{-1}^{1} K_{0}^{+}+\Pi_{-1}^{+} K_{0}^{1}\right)(E, \Lambda)$ in $j_{-1}^{\bar{M}}$ can be transformed to the expression $\left[\theta\left(\Delta^{2}-E_{+}^{2}\right)\left(\Pi_{1}^{1} K_{0}^{+}+\Pi_{1}^{+} K_{0}^{1}\right)\right](-E,-\Lambda)$, which cancels the analogous term with the arguments $(E,-\Lambda)$ in $j_{1}^{\bar{M}}$ after replacement $E \rightarrow-E$ in the integral in Eq. (55). Similar conclusions concern the term $\theta\left(\Delta^{2}-E_{+}^{2}\right)\left(\Pi_{1}^{1} K_{0}^{+}+\Pi_{1}^{+} K_{0}^{1}\right)$ in $j_{1}^{\bar{M}}$ and the terms, proportional to $M^{2}$, all of which contain unity matrix components.

\section{Appendix B. Analysis of partial multiparticle currents}

Here we briefly describe the asymptotic analysis of these partial contributions, using the methods and results developed earlier [18], with necessary modification due to asymmetry of the problem. To this end it is useful to express all the relevant quantities through the following functions

$$
\begin{aligned}
& \mathcal{N}_{m}=\rho_{m} N_{m+1 / 2} \equiv \rho_{m} N\left(E_{m+1 / 2}\right), \quad \mathcal{M}_{m}=\rho_{m} M_{m+1 / 2}, \\
& \overline{\mathcal{N}}_{m}=\rho_{m} \bar{N}_{m+1 / 2}, \quad \overline{\mathcal{M}}_{m}=\rho_{m} \bar{M}_{m+1 / 2}, \quad \tilde{f}_{m}=f_{m+1 / 2} .
\end{aligned}
$$


Let us now express the current spectral density in Eq. (50), $j_{0}+j_{1}+j_{-1} \equiv 2 j$, through the quantities introduced in Eq. (B.1) and put there $r_{1}=r_{2}=1 \quad\left(\rho_{m}=1\right)$, which leads to the expression for the current spectral density in the symmetric junction. Then we note that the derivation of the asymptotic expressions of the current in the case of symmetric junction [18] is performed by only using the analytical properties of the functions in Eqs. (B.1) at $r_{1}=r_{2}$ and the symmetries with respect to the permutation $E, \lambda \rightarrow-E,-\lambda$. Analysis shows that the functions for the asymmetric junction defined in Eqs. (B.1) have the same analytical properties and symmetries if we assume the simultaneous permutation $r_{1} \leftrightarrow r_{2}$. Therefore we conclude that to obtain the expression for the current in an asymmetric junction, one has to replace the current spectral density of a symmetric junction as follows:

$2 j\left\{r_{1}=r_{2}\right\} \rightarrow j\left\{r_{1}, r_{2}\right\}+\left(r_{1} \leftrightarrow r_{2}\right), \quad R_{1}=R_{2} \rightarrow R$.

This enables us, using the results of [18], to write down the final formula for multiparticle currents in an asymmetric junction,

$$
\begin{aligned}
I^{(n)}= & n\left(r_{1} r_{2}\right)^{n} \int_{-\infty}^{\infty} \frac{d \lambda}{2 \pi} \int_{\Delta-e V / 2}^{-\Delta+(n-1 / 2) e V / 2} \frac{d E}{2 e R} N_{1 / 2} N_{1 / 2-n} \\
& \times\left[\frac{1}{Z_{0}} \prod_{k=1}^{n-1} \frac{\bar{M}_{1 / 2-k}^{2}}{4 Z_{-k}}+\left(r_{1} \leftrightarrow r_{2}\right)\right]
\end{aligned}
$$

$Z_{0}=-\operatorname{det}\left(h_{0}+\Pi_{1}+\Pi_{-1}\right)$,

$Z_{m \gtrless 0}=-\operatorname{det}\left(h_{m}+\Pi_{m \pm 1}\right)$

(at $n=1$, the product in Eq. (B.2) is assumed to be unity).

Practical calculations using Eq. (B.2) require an appropriate choice of approximation for the determinants in Eqs. (B.3) and (B.4). These quantities can be expressed, using the recurrence (41) for $\Pi_{m}$, through the chain fractions that should be truncated at the $n$th step for the $n$-particle current,

$Z_{m}=\left|Z_{m}\right|^{2}$,

$z_{m>0}=\widetilde{h}_{m}-\frac{g_{m}^{2}}{\widetilde{h}_{m+1}-\frac{g_{m+1}^{2}}{\tilde{h}_{m+2}-\cdots}}, \quad z_{m<0}=\widetilde{h}_{m}-\frac{g_{m-1}^{2}}{\tilde{h}_{m-1}-\frac{g_{m-2}^{2}}{\hat{h}_{m-2}-\ldots}}$,

$z_{0}=\widetilde{h}_{0}-\frac{g_{0}^{2}}{\widetilde{h}_{1}-\frac{g_{1}^{2}}{\tilde{h}_{2}-\ldots}}-\frac{g_{-1}^{2}}{\widetilde{h}_{-1}-\frac{g_{-2}^{2}}{\tilde{h}_{-2}-\ldots}}, \quad \widetilde{h}_{m} \equiv h_{m}^{1}+h_{m}^{z}$.

\section{Appendix C. Evaluation of excess current}

The method of calculation of $I^{\text {exc }}$ is quite similar to that used in [18]. The basic idea of this method relies on the fact that only the energies of the order of $\Delta$ contribute into $I^{e x c}$, therefore at $e V \rightarrow \infty$ all spectral functions $M, \bar{M}$, and $\bar{N}$ with "shifted" energy $E+k e V, k \neq 0$, turn to zero, and the density of states $N(E+k e V)$ can be put to its limiting value (unity). This effectively truncates the recurrences (41) for $\Pi_{m}$ and enables us to write down $I^{e x c}$ as the integral over $E$ and $\lambda$ of the explicitly defined function. We will omit more detailed description of this procedure, which is rather cumbersome due to the junction asymmetry, and present only final results.

At arbitrary barrier strength $\gamma$, the integration over $\lambda$ can be performed analytically which leads to the following expression at $T=0$,

$I^{e x c}=\frac{1}{e R_{T}}\left(\int_{0}^{\Delta} d E j^{<}+\int_{\Delta}^{\infty} d E N j^{>}\right)$, where

$$
\begin{aligned}
& j^{<}=r_{1} r_{2} \bar{M}^{2} \sum_{i=1,2}\left(t_{i} \sqrt{t_{i}-c_{i}}\right)^{-1}, \quad t_{i}=\sqrt{c_{i}^{2}+b_{i}^{2}}, \\
& c_{i}=2\left(\tau^{2} E^{2}-1-\tau \bar{N} r_{i} E\right)+r_{1} r_{2}, \quad b_{i}=2 \tau E\left(2-r_{i}\right)-r_{1} r_{2} \bar{N} \\
& j^{>}=\sum_{i=1,2}\left(T_{i}-A_{-}\right)^{-1 / 2}\left(A_{+} / T_{i}+1\right)-2, \quad T_{i}=\sqrt{A_{-}^{2}+B_{i}^{2}}, \\
& A_{ \pm}=2\left(\tau^{2} E^{2} \pm 1\right) \pm r_{1} r_{2}(N-1), \quad B_{i}=2 \tau E\left[2+r_{i}(N-1)\right] .
\end{aligned}
$$

At large $\gamma \gg 1$, the second term in Eq. (C.1) dominates, and the integration can be done analytically, leading to Eq. (56).

In the regime of small dephasing, $\gamma \ll 1$, it is reasonable to first perform the integration over energy in the initial expression for $I^{\text {exc }}$ and then, introducing the transparency variable $D=r_{1} r_{2} /\left(\lambda^{2}+1\right)$, to express the excess current through its value for a single ballistic channel [46] averaged over the transparency distribution (19), in accordance with Eq. (18), which results in Eq. (58).

\section{References}

[1] Y.-J. Doh, J.A. van Dam, A.L. Roest, E.P.A.M. Bakkers, L.P. Kouwenhoven, S. De Franceschi, Science 309 (2005) 272.

[2] J. Xiang, A. Vidan, M. Tinkham, R.M. Westervelt, C.M. Lieber, Nat. Nanotechnol 1 (2006) 208.

[3] T.S. Jespersen, M.L. Polianski, C.B. Sørensen, K. Flensberg, J. Nygaard, New J. Phys. 11 (2009) 113025

[4] Y.-J. Doh, S.D. Franceschi, E.P.A.M. Bakkers, L.P. Kouwenhoven, Nano Lett. 8 (2008) 4098.

[5] L. Hofstetter, S. Csonka, J. Nygaard, C. Schönenberger, Nature 461 (2009) 960.

[6] T. Nishio, T. Kozakai, S. Amaha, M. Larsson, H.A. Nilsson, H.Q. Xu, G. Zhang, K. Tateno, H. Takayanagi, K. Ishibashi, Nanotechnology 22 (2011) 445701.

[7] H.A. Nilsson, P. Samuelsson, P. Caroff, H.Q. Xu, Nano Lett. 12 (2012) 228.

[8] S. Abay, H. Nilsson, F. Wu, H. Xu, C. Wilson, P. Delsing, Nano Lett. 12 (2012) 5622.

[9] S. Abay, D. Persson, H. Nilsson, H.Q. Xu, M. Fogelström, V. Shumeiko, P. Delsing, Nano Lett. 13 (2013) 3614.

[10] A. Kretinin, A. Das, H. Shtrikman, cond-mat./arXiv:1303.1410.

[11] S. Abay, D. Persson, H. Nilsson, Fan Wu, H.Q. Xu, M. Fogelström, V. Shumeiko, P. Delsing, cond.mat./arXiv:1311.1745.

[12] A.A. Golubov, M.Yu. Kupriyanov, E. Il'ichev, Rev. Mod. Phys. 76 (2004) 412.

[13] A. Bardas, D.V. Averin, Phys. Rev. B 56 (1997) R8518.

[14] A.V. Zaitsev, D.V. Averin, Phys. Rev. Lett. 80 (1998) 3602.

[15] E.N. Bratus', V.S. Shumeiko, G. Wendin, Phys. Rev. Lett. 74 (1995) 2110.

[16] D. Averin, A. Bardas, Phys. Rev. Lett. 75 (1995) 1831.

[17] J.C. Cuevas, A. Martín-Rodero, A. Levy Yeyati, Phys. Rev. B 54 (1996) 7366.

[18] E.V. Bezuglyi, E.N. Bratus', V.S. Shumeiko, Phys. Rev. B 83 (2011) 184517.

[19] K.K. Likharev, V.K. Semenov, IEEE Trans. Appl. Supercond. 1 (1991) 3.

[20] C.A. Hamilton, C.J. Burroughs, R.L. Kauts, IEEE Trans. Instrum. Meas. 44 (1995) 233.

[21] M.M.Th.M. Dierichs, P. Dieleman, J.J. Wezelman, C.E. Honingh, T.M. Klapwijk, Appl. Phys. Lett. 64 (1994) 921.

[22] E. Bartolomé, A. Brinkman, J. Flokstra, A.A. Golubov, H. Rogalla, Physica C 340 (2000) 93.

[23] M.Yu. Kupriyanov, A. Brinkman, A.A. Golubov, M. Ziegel, H. Rogalla, Physica C 326-327 (1999) 16

[24] A.I. Larkin, Yu.N. Ovchinnikov, in: D.N. Langenberg, A.I. Larkin (Eds.) Nonequilibrium Superconductivity, Elsevier, Amsterdam, 1986.

[25] M.Yu. Kupriyanov, V.F. Lukichev, Zh. Eksp. Teor. Fiz. 94 (1988) 139 [Sov. Phys JETP 67 (1988) 1163].

[26] R. Seviour, A.F. Volkov, Phys. Rev. B 61 (2000) 9273.

[27] E.V. Bezuglyi, V.S. Shumeiko, G. Wendin, Phys. Rev. B 68 (2003) 134506.

[28] P. Samuelsson, Phys. Rev. B 67 (2003) 054508.

[29] J. Börlin, W. Belzig, C. Bruder, Phys. Rev. Lett. 88 (2002) 197001.

[30] Yu.V. Nazarov, Superlatt. Microstruct. 25 (1999) 1221.

[31] W. Belzig, Yu.V. Nazarov, Phys. Rev. Lett. 87 (2001) 197006.

[32] J.A. Melsen, C.W.J. Beenakker, Physica B 203 (1994) 219.

[33] W. Belzig, A. Brataas, Yu.V. Nazarov, G.E.W. Bauer, Phys. Rev. B 62 (2000) 9726.

[34] P.W. Brouwer, C.W.J. Beenakker, Chaos, Solitons Fractals 8 (1997) 1249.

[35] L.E. Hasselberg, M.T. Levinsen, M.R. Samuelsen, Phys. Rev. B 9 (1974) 3757.

[36] E.V. Bezuglyi, A.S. Vasenko, E.N. Bratus', V.S. Shumeiko, G. Wendin, Phys. Rev. B 73 (2006) 220506;.

[37] E.V. Bezuglyi, A.S. Vasenko, E.N. Bratus', V.S. Shumeiko, G. Wendin, Supercond Sci. Technol. 20 (2007) 529.

[38] Enhanced conductance at $\Delta_{g}$ also appears in NN'S junctions, see A.F. Volkov, A.V. Zaitsev, T.M. Klapwijk, Physica C 210 (1993) 21.

[39] G. Johansson, E.N. Bratus', V.S. Shumeiko, G. Wendin, Phys. Rev. B 60 (1999) 1382.

[40] Å. Ingerman, G. Johansson, V.S. Shumeiko, G. Wendin, Phys. Rev. B 64 (2001) 144504. 
[41] P. Samuelsson, G. Johansson, Å. Ingerman, V.S. Shumeiko, G. Wendin, Phys Rev. B 65 (2002) 180514(R).

[42] J.C. Cuevas, J. Hammer, J. Kopu, J.K. Viljas, M. Eschrig, Phys. Rev. B 73 (2006) 184505.

[43] J. Kutchinsky, R. Taboryski, O. Kuhn, C.B. Sørensen, P.E. Lindelof, A. Kristensen, J. Bindslev Hansen, C. Schelde Jacobsen, J.L. Skov, Phys. Rev. B 56 (1997) 2932.
[44] R. Taboryski, J. Kutchinsky, J. Bindslev Hansen, M. Wildt, C.B. Sørensen, P.E. Lindelof, Superlattices Microstruct. 25 (1999) 829.

[45] T. Hoss, C. Strunk, T. Nussbaumer, R. Huber, U. Staufer, C. Schönenberger, Phys. Rev. B 62 (2000) 4079.

[46] V.S. Shumeiko, E.N. Bratus', G. Wendin, Fiz. Nizk. Temp. 23 (1997) 249 [Low Temp. Phys. 23 (1997) 181]. 